\title{
Biomechanical Comparison of Fixed- versus Variable-Angle Locking Screws for Distal Humerus Comminuted Fractures
}

\author{
Ali Nourbakhsh, MD, Adam G. Hirschfeld, MD*, Sravan Dhulipala, MD, William Hutton, $\mathrm{PhD}^{\dagger}$, \\ Timothy Ganey, PhD, Luis Lozada, MS, Daniel Schlatterer, MD, Gary Mark Lourie, MD
}

\author{
Department of Orthopedics, WellStar Atlanta Medical Center, Atlanta, GA, \\ ${ }^{*}$ Department of Orthopedics, MetroHealth Medical Center, Cleveland, $\mathrm{OH}$, \\ ${ }^{\dagger}$ Department of Orthopedics, Emory University School of Medicine, Atlanta, GA, \\ ${ }^{\ddagger}$ Department of Orthopedics, The Hand and Upper Extremity Center of Georgia, Atlanta, GA, USA
}

\begin{abstract}
Background: To compare the stability of fixed- versus variable-angle locking constructs for the comminuted distal humerus fracture (AO/OTA 13-A3).

Methods: Eight pairs of complete humeri harvested from eight fresh frozen cadavers were used for the study. We fixed the intact humeri using 2.7-mm/3.5-mm locking VA-LCP stainless steel distal humerus posterolateral (nine-hole) and medial (seven-hole) plates. An oscillating saw was used to cut a 1-cm gap above the olecranon fossa. The specimens were loaded in axial mode with the rate of $1 \mathrm{~mm}$ per 10 seconds to failure, and stress-strain curves were compared in each pair. The mode of failure was recorded as well as the load needed for 2- and 4-mm displacement at the lateral end of the gap.

Results: The stiffness of the constructs, based on the slope of the stress-strain curve, did not show any difference between the fixed- versus variable-angle constructs. Likewise, there was no difference between the force needed for 2- or 4-mm displacement at the lateral gap between the fixed- and variable-angle constructs. The mode of failure was bending of both plates in all specimens and screw pull-out in four specimen pairs in addition to the plate bending.

Conclusions: Our results did not show any difference in the biomechanical stability of the fixed- versus variable-angle constructs. There was not any screw breakage or failure of the plate-screw interface.
\end{abstract}

Keywords: Humerus, Fracture fixation, Bone screws

Comminuted extra-articular distal humerus fractures often result from high-energy trauma or low-energy trauma in the elderly. The comminution leads to instability of these fractures and surgical fixation is often required. For distal humerus fractures, dual plate fixation has been shown to be biomechanically superior to single plate fixa-

Received July 16, 2018; Revised August 21, 2018; Accepted August 22, 2018

Correspondence to: Gary Mark Lourie, MD

Department of Orthopedics, The Hand and Upper Extremity Center of Georgia, 980 Johnson Ferry Rd, NE, Suite 1020, Atlanta, GA 30342, USA

Tel: +1-404-255-0226, Fax: +1-678-539-6208

E-mail: gmlhtc@aol.com tion. ${ }^{1)}$ Locking plates are more stable in axial loading than the nonlocking constructs. ${ }^{2)}$ The two categories of locking plates are fixed-angle and variable-angle locking plates. In a variable-angle construct, the screw can be locked into the corresponding hole within a cone with an angle of $1^{\circ}$ to $15^{\circ}$ (unidirectional) and an overall variable arc of $30^{\circ 3}$ (Zimmer and Depuy Synthes). ${ }^{4)}$ In some fixation systems, there is a posterolateral plate with a distal phalange extending to the lateral side of the humerus. This allows lateral to medial screw placement. Contouring of this portion of the plate is also possible to create variability in the screw trajectory without altering its head relationship to the plate. Other available technology includes cutting a custom thread in the plate prior to screw insertion or us- 
Nourbakhsh et al. Variable- versus Fixed-Angle Locking Screws

Clinics in Orthopedic Surgery • Vol. 11, No. 3, 2019• www.ecios.org

ing a chrome screw, which can cut its own thread through a titanium plate hole. However, contouring the plate or making holes in it affects the biomechanical properties of the plate, which is not ideal. Also, lower-profile implants are preferred around the distal humerus to prevent skin irritation and soft tissue breakdown.

In a fixed-angle locking construct, the screws are inserted perpendicular to the screw hole, which might limit the amount of screw purchase into the fractured fragment. ${ }^{4)}$ Variable-angle constructs were designed to overcome this shortcoming. Additionally, variable-angle constructs are being used to avoid joint penetration and screw convergence, which might result in conflicting of the screws seen in fixed-angle constructs. ${ }^{3)}$

The stability of the variable-angle constructs has been tested in both distal femur ${ }^{4)}$ and distal radius models. ${ }^{5)}$ The purpose of our study was to compare the stability of fixed- versus variable-angle locking constructs for the comminuted distal humerus fracture (AO/OTA 13-A3). Since the use of variable-angle constructs are becoming more prevalent among orthopedic surgeons, the biomechanical soundness of them needs to be evaluated. To our knowledge, this is the first study comparing the fixed- and variable-angle constructs for distal humerus fractures. Additionally, most of the previously performed studies on other anatomic areas comparing these two constructs have used synthetic composite materials. ${ }^{4-6)}$

\section{METHODS}

Eight matched pairs of complete humeri harvested from eight fresh frozen cadavers were used for the study. The mean age of the cadavers was $71 \pm 13$ years (range, 45 to 88 years). There were four females and four males. Prior to fixation, the frozen specimens were thawed at room temperature for an average of 24 hours. Soft tissue was sharply dissected from the bone after 24 hours of thawing at room temperature. We used the $90^{\circ}$ fixation using 2.7- $\mathrm{mm} / 3.5-\mathrm{mm}$ locking VA-LCP stainless steel distal humerus posterolateral (nine-hole) and medial (sevenhole) plates (Depuy Synthes, West Chester, PA, USA). For consistency for each pair of specimens, we fixed the right humerus with a fixed-angle locking construct and the left humerus with a variable-angle construct. Fixation was initiated by putting the posterolateral plate over the posterior surface of the lateral column of the humerus as distally as possible, assuring that the distal end of the plate did not extend and the plate was completely sitting on the bone. A large reduction forceps was used to reduce the plate to the bone proximally and distally. Three $3.5-\mathrm{mm}$ bicortical screws were used at the most proximal screw holes of the plate and three fixed-angle (right side) or variable-angle (left side) screws were used at the most distal holes of the plate. The medial plate was subsequently fixed to the bone. For the fixed-angle locking screws, the guide was used, and for the variable-angle screws, the extreme angle of insertion $\left(15^{\circ}\right.$ from the mid-axis of the cone) was chosen (Fig. 1A). The anatomy of the distal humerus can be variable. The contour and shape of the plates that we used were the same for all the specimens. In many instances, we realized that insertion of the screw at certain angles might not provide adequate fixation. This was because we did not have enough bone to purchase at the angle and significant length of the screw would come out of the far cortex. We directed the variable-angle screws in an orientation to provide the best purchase into the bone. We matched the length of the screws for each pair of specimens (fixed vs. variable angle).

Osteotomy was performed after application of the plates in order to get a more anatomic fixation. An oscillating saw was used to cut a $1-\mathrm{cm}$ gap above the olecranon fossa. The upper edge of the olecranon fossa was referenced and a marking was made $1 \mathrm{~cm}$ above that line. From these points, two lines perpendicular to the axis of the bone were drawn. Both plates were protected while using the saw by thin metal objects. In order to be able to mount the construct in the biomechanical testing machine, we cut the proximal end of the humerus $5 \mathrm{~cm}$ above the proximal end of the posterolateral plate. All specimens were tested on the same day or the day after plate fixation. The constructs on the paired humeri were the same in regards to the length of the screws, the length and orientation of the nonlocking screws, and the position of the implants.

\section{Biomechanical Testing}

Sixteen specimens (eight pairs of humerii) were tested in axial loading on a material testing system (MTS) Mini Bionix testing machine (MTS, Eden Prairie, MN, USA). The fixed specimens we refrigerated for less than 24 hours prior to testing. The proximal end of the humerus was mounted into the machine. The distal end of the humerus was potted in bismuth dryable cement plastic pots. This would allow each humerus to be set in an identical position. Each humerus, in its pot, was mounted on the testing machine (Fig. 1B). The specimens were loaded in axial mode with the rate of $0.1 \mathrm{~mm} / \mathrm{sec}$ to failure, and the stiffness (slope of the initial linear region of the load-displacement curve) was compared in each pair. Figs. 2 and 3 show the load-displacement curves of the fixed- and variableangle constructs. The stiffness and mode of failure were 

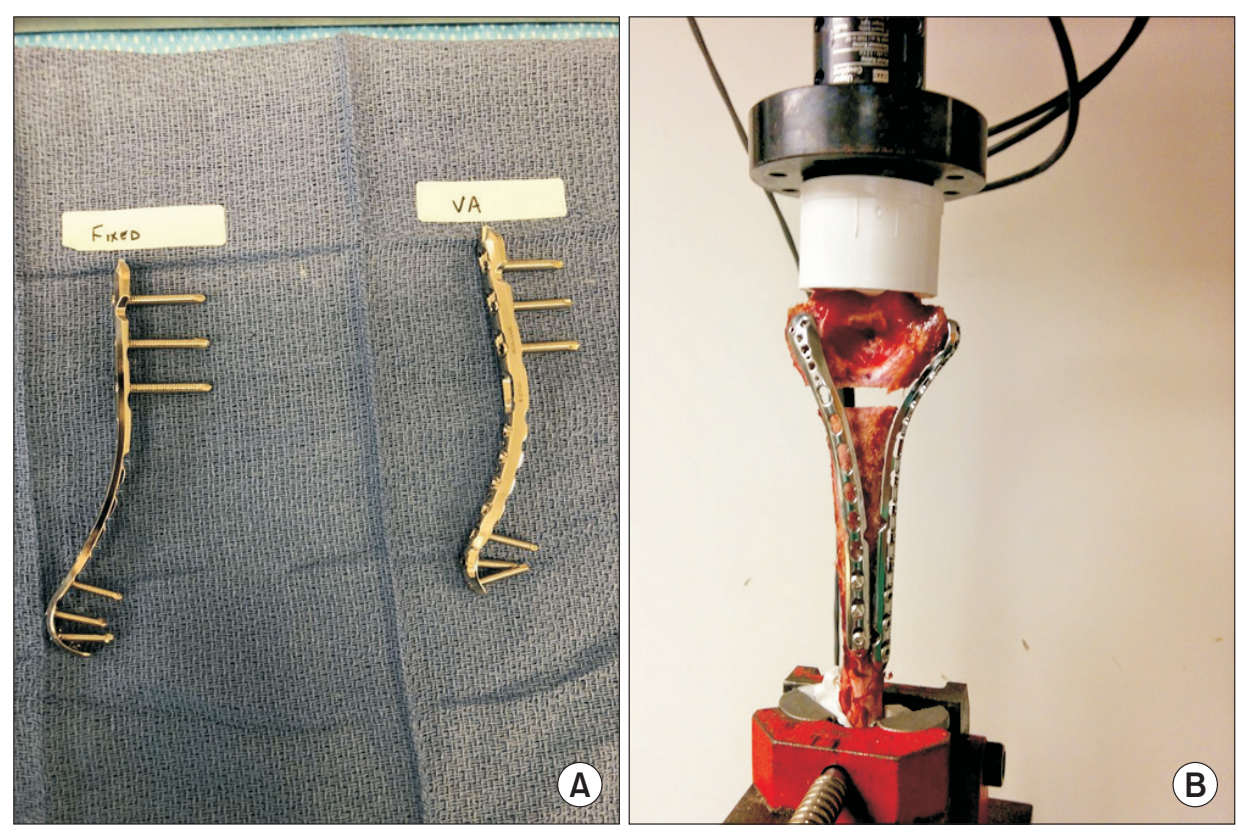

Fig. 1. (A) The distal and proximal configuration of the fixed- and variable-angle (VA) constructs. (B) Distal humerus dual plating is done and the specimen is mounted in the material testing system (MTS) Mini Bionix testing machine (MTS, Eden Prairie, MN, USA).

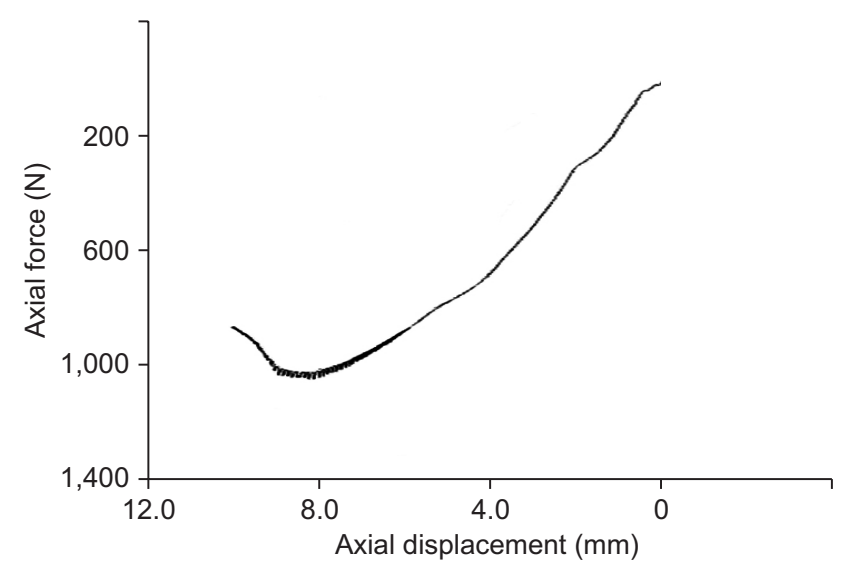

Fig. 2. The load-displacement curve of a fixed-angle construct.

recorded as well as the load needed for 2- and 4-mm displacement at the lateral end of the gap.

After testing, the specimens were evaluated radiologically by obtaining multiple views of the constructs to inspect the screws of both plates. We used IBM SPSS ver. 20.0 (IBM Corp., Armonk, NY, USA) for analysis. We used paired $t$-test to compare the stiffness, as well as the force needed for 2- and 4-mm displacement at the lateral end of the gap, between the fixed- and variable-angle constructs. We also compared the stability of the constructs between males and females and among different age groups using analysis of variance (ANOVA) and $t$-tests. A $p$-value less than 0.05 was considered significant. No institutional review board approval was necessary for this cadaveric study.

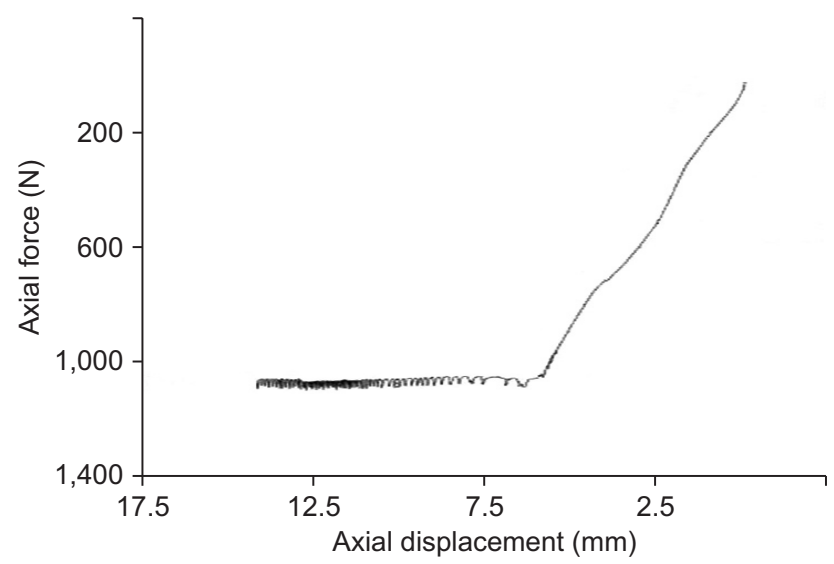

Fig. 3. The load-displacement curve of a variable-angle construct.

\section{RESULTS}

There was no difference between the age of males and females ( 73 vs. 71 years, $p=0.770$ ). The stiffness of the constructs did not show any difference between the fixed- and variable-angle constructs ( $123.48 \pm 46.42$ vs. $120.84 \pm 59.98$ $\mathrm{N} / \mathrm{mm}, p=0.923)$. Likewise, there was no difference between the fixed-and variable-angle constructs in the force needed for $2-\mathrm{mm}(620.63 \pm 243.02$ vs. $518.75 \pm 189.17 \mathrm{~N}$, $p=0.365)$ or $4-\mathrm{mm}(234.16 \pm 104.72$ vs. $276.53 \pm 123.67 \mathrm{~N}$, $p=0.941)$ displacement at the lateral gap. There was also no difference in the stiffness and force needed for 2- and 4-mm displacement among different age groups. There was a wide age range among our cadavers. However, these variables were all significantly higher in male cadavers than 
Nourbakhsh et al. Variable- versus Fixed-Angle Locking Screws

Clinics in Orthopedic Surgery • Vol. 11, No. 3, 2019• www.ecios.org

Table 1. Stiffness and Force Needed for 2- and 4-mm Displacement among Male and Female Cadavers

\begin{tabular}{lccc}
\multicolumn{1}{c}{ Variable } & Male & Female & $p$-value \\
\hline Stiffness (N/mm) & $151.54 \pm 33.27$ & $92.76 \pm 51.74$ & 0.017 \\
\hline Force needed for 2-mm displacement (N) & $681.25 \pm 220.03$ & $458.13 \pm 154.64$ & 0.034 \\
\hline Force needed for 4-mm displacement (N) & $903 \pm 210.97$ & $521.43 \pm 77.55$ & 0.001 \\
\hline
\end{tabular}

Values are presented as mean \pm standard deviation.

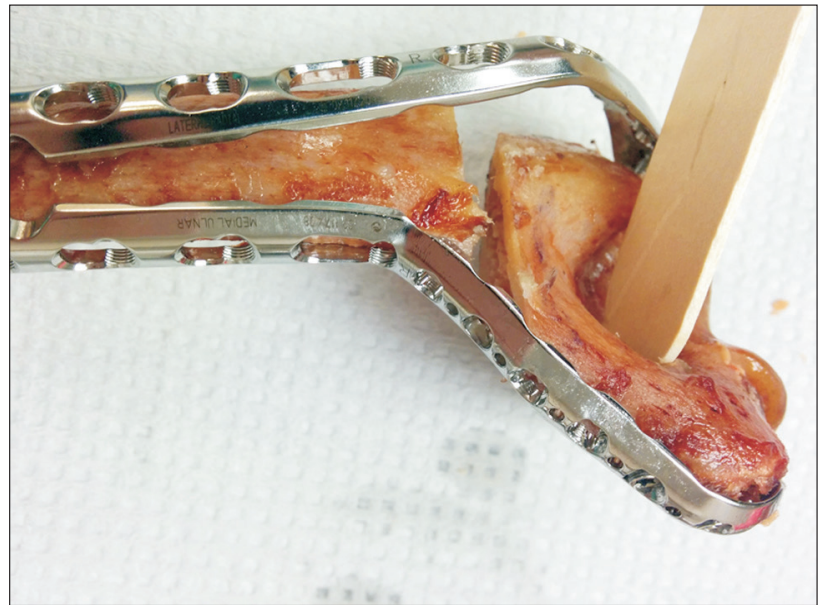

Fig. 4. The posterolateral plate bent after biomechanical testing.

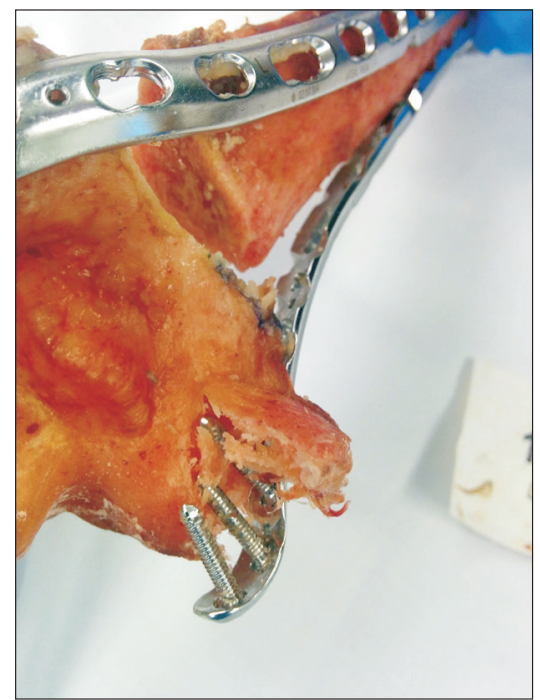

Fig. 5. The locking screws at the distal end of the medial plate broke out of the cortex.

female cadavers (four specimens in each group) (Table 1).

The mode of failure was bending of both plates in all specimens (Fig. 4). In four pairs of specimens, the lock-
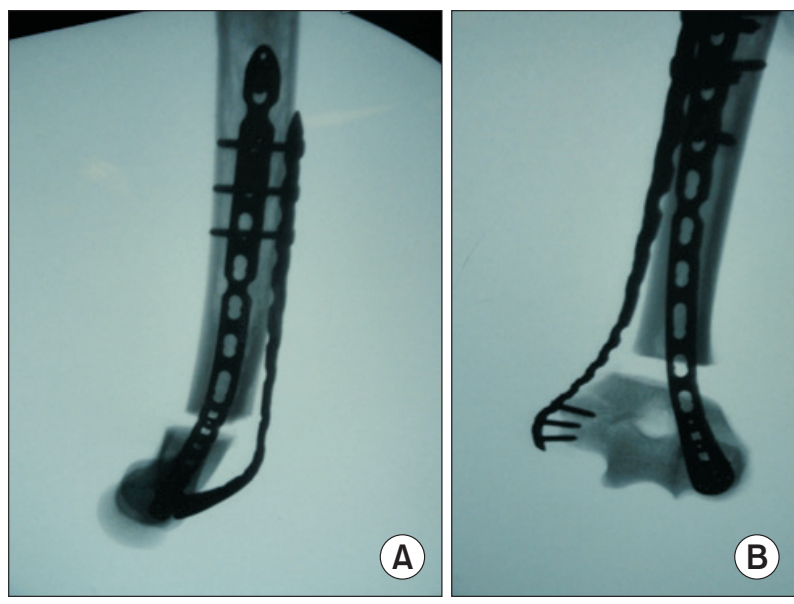

Fig. 6. (A) The lateral X-ray of the distal humerus shows the bent posterolateral plate. (B) The anteroposterior view of the distal humerus shows the distal locking screws of the medial plate breaking out of the cortex.

ing screws broke out of the cortex in addition to bending of the plate. In two pairs of specimens, both the fixedand variable-angle constructs within each matched pair failed in the same manner. The distal fixed- and variableangle locking screws of the medial plate pulled out of the cortex (Fig. 5). In the other two matched pairs, the mode of failure was different: in one pair, the fixed-angle locking screws of the posterolateral plate on one side and the variable-angle locking screws of the medial plate on the other side pulled out of the cortex; in the last pair, the opposite of this happened. On inspection and imaging, there was no screw breakage or plate-screw interface failure (Fig. $6)$.

\section{DISCUSSION}

In our study, the stiffness of the variable- versus fixed-angle screws were evaluated in axial load. Our results showed no difference between the two screws. The screw hole was the same for either a fixed- or variable-angle screw. Un- 
Nourbakhsh et al. Variable- versus Fixed-Angle Locking Screws

Clinics in Orthopedic Surgery • Vol. 11, No. 3, $2019 \bullet$ www.ecios.org

like a traditional fixed-angle locking plate, the fixed-angle screws also thread into the four threaded flanges within each screw hole. In the Depuy Synthes system, the fixedangle locking screw head has a conical shape with threads that lock within the threaded hole. The pitch of the screw head and the body of the screw are identical to prevent compression. In the variable-angle screw system, the screw head is spherical and threaded. It is screwed inside the pilot hole in four threaded flanges. ${ }^{3)}$ The osteotomy was made to simulate unstable comminution by cutting 1 $\mathrm{cm}$ of bone above the olecranon fossa. Additionally, our model included a bony gap because in non-gap models, axial deformation results in contact between the cortices of proximal and distal fragments, which can confound the results. ${ }^{6}$ Dual plate fixation was used since it has been proven to be stronger than single plate fixation. ${ }^{1)}$ Dual plate fixation might be indicated in cases of osteoporosis and comminution. We used locking constructs since they have been shown to provide better stability for distal humerus fractures compared to nonlocking constructs. Schwartz et al. ${ }^{8)}$ compared $180^{\circ}$ versus $90^{\circ}$ dual plate constructs for segmental intra-articular distal humerus fractures. They concluded that both constructs provided the same mechanical stiffness. Same results were found by Penzkofer et al. ${ }^{9)}$ We chose to use three distal screws in this model. Hart et al. ${ }^{10)}$ did a biomechanical study that compared dual plating of the distal humerus with either one or three screws in the distal ulna column for AO/OTA A3 type fractures. This study showed that the constructs with three distal screws had a higher stiffness in axial loading and bending.

Significant compressive and shear forces occur at the elbow joint. ${ }^{11)}$ The joint reaction force varies in different elbow positions: ${ }^{12)}$ it is greatest between $0^{\circ}$ and $30^{\circ}$ of flexion and is larger in pronation than supination. The direction of the joint reaction force changes with the angle of flexion, moving more posteriorly with elbow flexion. ${ }^{11)}$ The highest magnitude of compressive forces on the distal humerus during elbow range of motion is in line with the long axis of the humerus at $15^{\circ}$ of elbow flexion. At $0^{\circ}$ and $30^{\circ}$ of flexion, it moves $15^{\circ}$ posterior and anterior to the long axis of humerus, respectively. ${ }^{13)}$ In our study, we tested the specimens in axial load in line with the long axis of the humerus to evaluate the highest compressive force on the distal humerus during elbow range of motion. The average torque created at the elbow joint can be as high as 68.65 $\mathrm{N}-\mathrm{m}$ with the elbow at $90^{\circ}$ of flexion ${ }^{14)}$ and $8.83 \mathrm{~N}-\mathrm{m}$ in extension. Active stability of the elbow joint is maintained by the action of muscles that provide joint compressive forces. ${ }^{15,16)}$ It has been proven that the compressive force across the elbow joint is almost eight times the weight held by the outstretched hand. ${ }^{17)}$ This force might increase to three times the body weight during weight lifting. ${ }^{18)}$ This force peaks at early flexion during the elbow range of motion. During dressing and eating activities, the joint reactive force reaches $300 \mathrm{~N}$ and it increases to $1,700 \mathrm{~N}$ when rising from a chair. The joint reactive force of the elbow is $32 \mathrm{~N}$ in flexion and $107 \mathrm{~N}$ in extension due to the shorter lever arm of the triceps compared to biceps. ${ }^{17)}$ During the early postoperative period, protected range of motion of the elbow is recommended to prevent joint stiffness, which is one of the most common complications after distal humerus fractures. Also, simple activities of daily living exert substantial compressive force across the elbow joint. Considering the limited number of specimens, we decided to measure the stiffness of the aforementioned constructs in axial loading.

Our results did not show any difference between the biomechanical stability of the fixed-and variable-angle constructs. There was not any screw breakage or failure of the plate-screw interface. This proves the soundness of the variable-angle construct in distal humerus fracture where fragment-specific fixation seems crucial. In a biomechanical study, Hart et al. ${ }^{10)}$ showed that the use of variableangle constructs can compensate for the suboptimal plate positioning in unstable distal radius fractures.

The stability of the constructs was higher in male cadaveric specimens, which might be due to higher bone density in that group. The female cadavers were not older than the males. The mode of failure in all our constructs was plate bending. This was probably due to the design of the plates.

In an extra-articular femur fracture model, Otto et al. ${ }^{4)}$ tested the axial stability of a noncontact bridging (NCB) polyaxial locking plate (Zimmer), POLYAX plate (Biomet), and fixed-angle less invasive stabilization system (LISS; Synthes). The NCB plates have a diagonal threehole pattern. Offset holes can be used for screw placement around the prosthesis. Polyaxial screws $\left(30^{\circ}\right.$ cone) can be used with the use of locking caps that are threaded into the plate holes. ${ }^{19)}$ POLYAX system allows screws to be locked into the plate. Multiple screw options can be used including polyaxial or fixed locking and nonlocking screws within a $30^{\circ}$ cone of angulation. ${ }^{20)}$ They used 25 synthetic femurs and divided them into five groups. Constructs were tested under axial loading and loaded to failure with a $5 \mathrm{~mm} / \mathrm{min}$ displacement rate. The mode of failure for LISS and POLYAX constructs was plastic deformation of the plate, while it was intra-articular lateral condyle fracture in the NCB plates. There was no difference between 
Nourbakhsh et al. Variable- versus Fixed-Angle Locking Screws

Clinics in Orthopedic Surgery • Vol. 11, No. 3, 2019•www.ecios.org

the parallel and crossed polyaxial constructs. In regards to the stiffness, there was no difference between the LISS and $\mathrm{NCB}$, and both of them were higher than the POLYAX. They concluded that polyaxial locking constructs are biomechanically sound since there were no failures of either polyaxial screw-plate interface. ${ }^{4)}$ Same results were reported in a different study by Wilkens et al. ${ }^{21)}$

Stanbury et al. $^{5}$ tested the stability of AO-C3 distal radius fractures fixed by fixed- versus variable-angle locking constructs in axial compression using the synthetic composites. They evaluated the articular step-off and loadto-failure data. They concluded that variable-angle and fixed-angle volar locked fixation of unstable intra-articular distal radius fractures provided high load to failure measurements without loss of stiffness or articular step-off compared to fixed-angle constructs.

The limitations of our study are as follows: (1) The variable- and fixed-angle locking screws were inserted in the same method used in the operating room and the operator error is an inevitable part of this technique. (2) As the first study on variable- versus fixed-angle constructs on the distal humerus fractures, we evaluated the comminuted distal extra-articular humerus fractures. However, intra-articular fractures need to be studied in the future studies. (3) We did not measure the stability of individual screws in the fixed- and variable-angle locking constructs. It was assumed that the evaluation of the construct as a whole is more relevant to the clinical application. (4) For more consistency and decreasing differences between the two groups, we used the fixed-angle screws on the right side and variable-angle screws on the left humeri. If the majority of subjects were right-handed and, therefore, had subtly greater bone density of that limb, this could skew the results. However, even in that situation, we should have seen superiority of the fixed-angle screws. This might at least prove the noninferiority of the variableangle screws compared to the fixed-angle screws in axial loading. (5) We used paired humeri from one cadaver to obviate the need for densitometry of the bone, but the study would have been improved if quantification of bone density of the specimens could have been done. (6) There were limitations based on the number of specimens and the multitude of plate placement and locking technology options available. (7) Finally, we did not test the constructs in torsion, bending, or cyclical loading.

Summarily, this study (linear load to failure) showed that there is no difference between the stiffness of the fixed- and variable-angle locking constructs in $90^{\circ}$ dual plating of the comminuted extra-articular distal humerus fractures. Further studies are necessary to study these constructs in other types of distal humerus fractures including intra-articular patterns. Cyclical testing would also be worthwhile.

\section{CONFLICT OF INTEREST}

This study was funded by Depuy-Synthes in the amount of US $\$ 102,680$ (grant no. 11934). The funder had no role in the design, collection, analysis or interpretation of this study. No other potential conflict of interest relevant to this article was reported.

\section{ACKNOWLEDGEMENTS}

This study was funded by Depuy-Synthes in the amount of US \$102,680 (grant no. 11934).

\section{REFERENCES}

1. Scolaro JA, Hsu JE, Svach DJ, Mehta S. Plate selection for fixation of extra-articular distal humerus fractures: a biomechanical comparison of three different implants. Injury. 2014;45(12):2040-4.

2. Egol KA, Kubiak EN, Fulkerson E, Kummer FJ, Koval KJ. Biomechanics of locked plates and screws. J Orthop Trauma. 2004;18(8):488-93.

3. Cronier P, Pietu G, Dujardin C, Bigorre N, Ducellier F, Gerard R. The concept of locking plates. Orthop Traumatol Surg Res. 2010;96(4):S17-36.

4. Otto RJ, Moed BR, Bledsoe JG. Biomechanical comparison of polyaxial-type locking plates and a fixed-angle locking plate for internal fixation of distal femur fractures. J Orthop
Trauma. 2009;23(9):645-52.

5. Stanbury SJ, Salo A, Elfar JC. Biomechanical analysis of a volar variable-angle locking plate: the effect of capturing a distal radial styloid fragment. J Hand Surg Am. 2012;37(12):2488-94.

6. Cullen AB, Curtiss S, Lee MA. Biomechanical comparison of polyaxial and uniaxial locking plate fixation in a proximal tibial gap model. J Orthop Trauma. 2009;23(7):507-13.

7. Hungerer S, Wipf F, von Oldenburg G, Augat P, Penzkofer R. Complex distal humerus fractures-comparison of polyaxial locking and nonlocking screw configurations: a biomechanical study. J Orthop Trauma. 2014;28(3):130-6. 
8. Schwartz A, Oka R, Odell T, Mahar A. Biomechanical comparison of two different periarticular plating systems for stabilization of complex distal humerus fractures. Clin Biomech (Bristol, Avon). 2006;21(9):950-5.

9. Penzkofer R, Hungerer S, Wipf F, von Oldenburg G, Augat P. Anatomical plate configuration affects mechanical performance in distal humerus fractures. Clin Biomech (Bristol, Avon). 2010;25(10):972-8.

10. Hart A, Collins M, Chhatwal D, Steffen T, Harvey EJ, Martineau PA. Can the use of variable-angle volar locking plates compensate for suboptimal plate positioning in unstable distal radius fractures? A biomechanical study. J Orthop Trauma. 2015;29(1):e1-6.

11. Fornalski S, Gupta R, Lee TQ. Anatomy and biomechanics of the elbow joint. Tech Hand Up Extrem Surg. 2003;7(4):168-78.

12. Halls AA, Travill A. Transmission of pressures across the elbow joint. Anat Rec. 1964;150:243-7.

13. Amis AA, Dowson D, Wright V, Miller JH. The derivation of elbow joint forces, and their relation to prosthesis design. J Med Eng Technol. 1979;3(5):229-34.

14. Laksanacharoen S, Wongsiri S. Design of apparatus to study human elbow joint motion. In: IEEE EMBS Asian-Pacific Conference on Biomedical Engineering; 2003; Kyoto, Japan.
15. Dunning CE, Zarzour ZD, Patterson SD, Johnson JA, King GJ. Muscle forces and pronation stabilize the lateral ligament deficient elbow. Clin Orthop Relat Res. 2001;(388):118-24.

16. Shahid MK, Fletcher M, Robati S, Pemmaraju G. The biomechanical forces that act on the elbow joint. EC Orthop. 2015;1(1):1-11.

17. Nordin M, Frankel VH. Basic biomechanics of the musculoskeletal system. 4th ed. Philadelphia, PA: LWW; 2012. 352.

18. An KN, Hui FC, Morrey BF, Linscheid RL, Chao EY. Muscles across the elbow joint: a biomechanical analysis. J Biomech. 1981;14(10):659-69.

19. Zimmer. $\mathrm{NCB}^{\circledR}$ periprosthetic femur plate system [Internet]. Warsaw, IN: Zimmer; 2014 [cited 2019 Jun 15]. Available from: http://www.zimmer.com/medical-professionals/products/trauma/ncb-periprosthetic-femur.html.

20. Biomet. POLYAX periarticular plating system [Internet]. Switzerland: Biomet; 2014 [cited 2019 Jun 15]. Available from: https://www.yumpu.com/en/document/view/24185478/ polyax-distal-femoral-locked-plating-system-productbiomet.

21. Wilkens KJ, Curtiss S, Lee MA. Polyaxial locking plate fixation in distal femur fractures: a biomechanical comparison. J Orthop Trauma. 2008;22(9):624-8. 